NASA Technical Memorandum 100843

\title{
Application of Advanced Computational Technology to Propulsion CFD
}

(MASA-Ty-100843) ARELICATICY OF ADVAXCED CCAROTATICHAL TECHULCGY IC EECEULSIOA.CFD (MASA) $21 \mathrm{P}$

John R. Szuch

Lewis Research Center

Cleveland, Ohio

Prepared for the

Symposium on Advances and Trends in Computational Structural Mechanics and Fluid Dynamics

Washington, D.C., October 17-19, 1988 
APPLICATION OF ADVANCED COMPUTATIONAL TECHNOLOGY TO PROPULSION CFD

John R. Szuch

National Aeronautics and Space Administration

Lewis Research Center

Cleveland, Ohio 44135

\section{ABSTRACT}

The Internal Fluid Mechanics Division of the NASA Lewis Research Center is combining the key elements of computational fluid dynamics, aerothermodynamic experiments, and advanced computational technology to bring internal computational fluid mechanics (ICFM) to a state of practical application for aerospace propulsion system design. This paper presents an overview of efforts underway at NASA Lewis to advance and apply computational technology to ICFM. These efforts include the use of modern, software engineering principles for code development, the development of an AI-based user-interface for large codes, the establishment of a high-performance, data communications network to link ICFM researchers and facilities, and the application of parallel processing to speed up computationally-intensive and/or time-critical ICFM problems. A multi-stage compressor flow physics program is cited as an example of efforts to use advanced computational technology to enhance a current NASA Lewis ICFM research program.

\section{INTROOUCTION}

The development of advanced aerospace propulsion systems, that must be lighter, more durable, more efficient, and cost-competitive, demands increased understanding of all of the physical phenomena (e.g., coupled fluid and structural mechanics with three-dimensional viscous flows, turbulence, shockboundary layer interactions, combustion, heat transfer, multi-stage rotating machinery, flexible structures, high-temperature materials) that determine performance and life. 
The National Aeronautics and Space Administration is committed to carrying out research and technology programs to acquire basic understanding of the fundamental physics in propulsion systems and to build this understanding into numerical simulations that can be used to identify promising regions of propulsion system "design-space" for commercial exploitation.

As revolutionary changes take place in the designs of propulsion system components, the need for high-fidelity computer simulations becomes more acute because past experience and data cannot be extrapolated with confidence. Also, it may be prohibitively expensive to fabricate, assemble, and test each new concept and design under realistic conditions. In some cases (e.g., National Aerospace Plane), experimental facilities might not even exist to test new component and system designs.

The need exists, therefore, for computer simulations that can be used as "numerical test cells." In the case of computational fluid dynamics (CFD), however, very few simulation codes accurately portray the three-dimensional flow physics that takes place within real propulsion systems - either because of a lack of understanding of the fundamental physics or a lack of computing power (speed and/or memory) to solve the model equations in a timely fashion. As a result, propulsion system development remains a heuristic process, involving the use of highly empirical models and extensive hardware testing.

As progress is made in gaining a fuller understanding of the complex fluid dynamics in propulsion systems, we need to match that understanding with sufficient computational capability to allow the practical use of internal computational fluid mechanics (ICFM) codes as engineering and design tools. This implies numerical solutions and presentation of results to the analyst in minutes or hours, rather than days or weeks. Also, the scope of the simulation task must, in many instances, go beyond the calculation of only the fluid 
behavior in a single component to include the interactions of fluid and structural mechanics (i.e., aeroelasticity) and the interactions of various components in the overall propulsion system. This represents a tremendous computational challenge that will only be met through continued advances in (1) computer science and technology, and (2) the ability of the user community to exploit those advances.

This paper presents an overview of efforts underway at NASA Lewis to develop, adapt, and apply computational technology to propulsion CFD. These efforts include:

(1) The development of PROTEUS, a three-dimensional Navier-Stokes testbed flow solver. PROTEUS represents a novel approach to CFD code development that features a multi-disciplinary development team, employing modern software engineering techniques. The emphasis is on creating a user-friendly code that can be tailored to a variety of propulsion applications.

(2) The development of PROTAIS, an intelligent user-interface to the PROTEUS code. PROTAIS is a knowledge-based system that is being developed in collaboration with the PROTEUS code developers. The goal is to provide computer-based guidance and assistance to both expert and novice users of the PROTEUS code.

(3) The establishment of a high-performance, data communications network (ERBNET) that provides researchers with access to a wide spectrum of computing facilities, including local (departmental), centralized (Lewis-wide), and remote (e.g., Numerical Aerodynamic Simulator at NASA Ames) computer systems.

(4) In-house and sponsored research and development activities aimed at exploiting PARALLEL PROCESSING architectures for propulsion CFD. These activities range from multi-tasking of codes on the CRAY X/MP supercomputer to the buildup and testing of the HYPERCLUSTER, an experimental parallel processing 
workstation. Hardware and software strategies are being sought that can deliver fast, interactive, and affordable computing to the CFD researcher.

(5) Focused efforts to integrate and apply the aforementioned technologies and capabilities to enhance current and future CFD research programs. A proposed multi-stage compressor flow physics program is cited as an example. ICFM RESEARCH THRUSTS

The Internal Fluid Mechanics Division (IFMD) at NASA Lewis is combining the key elements of computational fluid dynamics, aerothermodynamic experiments, and advanced computational technology to bring internal computational fluid mechanics (ICFM) to a state of practical application for aerospace propulsion system design [1].

By focusing on specific portions of the propulsion system, IFMD researchers are working to isolate and study the dominant phenomena in order to gain the understanding needed to develop an accurate, predictive capability. As shown in Fig. 1, the ICFM program is organized with Inlets, Turbomachinery, and Chemical Reacting Flows as the main research thrusts.

The development of accurate, predictive propulsion CFD codes requires a close coupling of the numerical code development work and fundamental physical experiments. As shown in Fig. 2, each discipline is dependent on the other for information and guidance. The insights gained through fundamental physical experiments form the basis for new mathematical models and codes. The resultant codes must be tested by comparison of analytical and experimental data, often leading to new test requirements.

While the speed and memory capacity of computers have increased dramatically over the past 40 years, computer technology still remains a limiting factor in the quest to use high-fidelity computer simulations as tools in the 
propulsion system design process. Code developers must determine the appropriate level of model detail that will, when combined with available computational power, satisfy the needs of the intended application(s).

As an example, consider the range of computational tools envisioned for turbomachinery design and analysis. Figure 3 shows the relative levels of complexity of these tools, ranging from the unaveraged Navier-Stokes equations down to quasi-one-dimensional equations. The aggressive design of multi-stage turbomachines for the next century's propulsion systems will utilize complex geometries with endwall contouring and swept blading. Resulting flow fields will be highly three-dimensional with unsteady rotor-stator interactions and large secondary flows. It's clear that three-dimensional, unsteady, viscous codes will be needed to describe the physics. The same conclusions can be drawn for the other propulsion system components, as well. However, the use of high-fidelity ICFM codes requires a great deal of computer power. Figure 4 indicates that it will take a 10 Gword/100 Mflop machine to obtain a timeaccurate, Reynolds-averaged flow field solution, for a hypersonic inlet, in $1 \mathrm{CPU} \mathrm{hr}$. In a design process, where repetitive running of codes is required to perform sensitivity studies and parameter optimizations, it will require computers that have 100 gigaword in-core memories and Tflop speeds. Until such machines are readily available for propulsion system analysis, system developers will be forced to continue using empirical models. Therefore, an important byproduct of the efforts to develop more accurate, two- and three-dimensional codes is the building up of analytical and experimental databases that can be used to extend the range of the empirical models.

\section{APPLICATION OF NEW COMPUTATIONAL TECHNOLOGY}

While supercomputer speed is critical, there are many other computer technologies that can contribute to making ICFM practical for propulsion system 
design. As shown in Fig. 5, the code development process can also benefit from advances being made in software engineering, expert systems, networks, and parallel processing. If used properly, these technologies can be combined to form an integrated, cooperative environment for conducting ICFM analyses and experiments. The resulting databases, codes, and insights can, in turn, form the basis for a more confident, more aggressive design methodology.

The following paragraphs describe efforts underway to develop and demonstrate advanced computational technologies for propulsion CFD.

Software Engineering (PROTEUS)

Numerous efforts in CFD consist of modifying existing programs to handle geometries and flow regimes of current interest to the researcher. Unfortunately, nearly all nonproprietary programs were started as research projects with emphasis on demonstrating a numerical algorithm rather than ease of use or modification. The end result is usually a haphazard collection of poorly documented, extensively patched code that is difficult to understand and which may not even work under certain conditions.

Current efforts are directed at minimizing the above problems by standardizing the structure of newer codes. One such code is PROTEUS, a generalpurpose, three-dimensional Navier-Stokes flow solver. By rigorously adhering to the software engineering principles that are shown in Fig. 6, the PROTEUS developers are producing a code that has a consistent, clear structure and is easy to understand and modify.

Figure 7 illustrates the development cycle for the PROTEUS code. One of the more unusual aspects of the PROTEUS development has been the team approach to implementation. By specifying each program module in terms of its function and interfaces, each member of the multi-disciplinary development team has been able to independently code modules while treating the other modules as "black 
boxes." This approach has permitted programming assignments to be made on the basis of individual skills and experience and has led to a more timely implementation and testing of modules.

From the beginning of the PROTEUS project, the developers recognized the critical importance of documentation. Internal program documentation is provided by the liberal use of comment statements and header sections for each module. External documentation is provided by the creation of dictionaries that define input variables, common block variables, default variables, and module functions.

\section{Artificial Intelligence (PROTAIS)}

The application of software engineering principles to the PROTEUS development represented a big step toward making large CFD codes user-friendly. That effort has also provided a stimulus to researchers at NASA Lewis to investigate the use of artificial intelligence (AI) technology as an additional aid in developing and using such codes. The NASA Lewis effort attempts to capture the expertise gained by code developers in an "intelligent interface" that will allow others to make more effective use of the codes.

The interface is initially being developed to support the PROTEUS code and, as such, has been named PROTAIS to denote the introduction of AI techniques to PROTEUS [2]. As shown in Fig. 8, the PROTAIS system is intended to provide help and advise for both expert and novice users of PROTEUS. It features a constantly growing knowledge base that reflects the experience and knowledge gained as the PROTEUS code is developed and run under a variety of conditions.

The PROTAIS development is being done on a Symbolics workstation (Fig. 9). Work, to date, has concentrated on developing expert user facilities that automate many of the tedious and time-consuming aspects of setting up and running 
PROTEUS. The user can query the interface to obtain information on running the code including definitions and status of existing code configurations (i.e., master sets, problem sets, ranges of input variables and their default values, cases run and results obtained, and connected computer systems and their proto(cols). At this writing, testing of the software design is being carried out in a stand-alone mode (simulating the interface to PROTEUS) on the Symbolics workstation. Later this year, local-area network facilities (see description of ERBNET) will be used to connect the interface, installed on general-purpose engineering workstations, to the PROTEUS code, running on a mainframe computer. The interface will then undergo extensive testing and evaluation by the PROTEUS developers.

Future refinements to the PROTAIS interface will be aimed at novice users of the code, shielding those users from the details of the code and the environment under which it is running (i.e., computers, operating systems, networks, etc.). Rules (or other knowledge representations) used by CFD experts will be added to the interface to guide the novice user in effectively using the code.

\section{Networks (ERBNET)}

In 1985, work began on the design of a local-area network (ERBNET) that would allow ICFM researchers to access and communicate between various terminals, computers, and workstations located in the Engine Research Building (ERB) complex. The network would also provide connectivity and high-speed data communications between the local computers, centralized mainframe computers, and remote computers such as the NAS.

In designing the ERBNET system, special attention was given to system adaptability, flexibility, and growth potential. The decision was made to use proven and available technology (Ethernet communications and TCP/IP networking 
protocols) to reduce cost, allow rapid implementation, and permit modular $\cdots$ upgrades.

Figure 10 shows a block diagram representation of the ERBNET configuration. A baseband Ethernet cable was installed to provide high-speed (10 Mbits/sec) connections between local computers and workstations. A utility band on the Lewis-wide broadband cable (LINK) was used to interconnect ERBNET with computers in the central Research Analysis Center (RAC) and to provide user access to the NAS facilities through the NASA Program Support Communications Network (PSCN). Figure 11 shows one of the Silicon Graphics IRIS (Integrated Raster Imaging System) devices that is being used as a NAS workstation. The IRIS is capable of transforming and displaying three-dimensional data at rates exceeding 50000 points per second.

ERBNET continues to be expanded to provide additional capabilities to NASA Lewis researchers. ERBNET is being tied into several off-site networks to allow sharing of resources and information with university and industrial researchers. Additional equipment is being added to the network, including additional high-performance graphics workstations. Work is underway to develop graphics and scientific database software that will facilitate the interchange and display of data across the breadth of ERBNET devices.

Parallel Processing

Advancements in solid-state electronics and circuit design technology produced nearly linear increases in computer speeds from 1950 to 1970 . Since that time, computer manufacturers have had to look toward new computer architectures (e.g., vector and parallel processors) to gain additional computing speed. A modern supercomputer will have 2 to 8 , bus-connected, vector processors with shared memory. When run in a parallel processing mode, these supercomputers 
achieve benchmark speeds of 100 to 300 Mflops. As the pursuit of Tflop computers continues, the introduction of new, highly-parallel architectures poses significant challenges (and opportunities) for ICFM algorithm and code developers who must devise methods for harnessing the power of these machines.

The calculation of three-dimensional, unsteady flow fields within multistage compressors and turbines is a prime candidate for parallel processing because of (1) the long run times that will be required to perform the multistage calculations and (2) the many levels of parallelism that do exist in current multi-stage algorithms. Mulac, et al., have demonstrated a technique for using the parallel processing capabilities of today's supercomputers for turbomachinery CFD [3]. An "average-passage" model that can compute flow fields in up to 20 blade rows (10 stages) was developed. As shown in Fig. 12, the average-passage model has been tested on a 4-processor CRAY X-MP with each blade row of a 2-stage turbine assigned to a separate CPU. The continuity, momentum, and energy equations for each blade row are computed in parallel. The effects of body forces (the other blade rows) on each blade row solution are handled with stress-like terms. While the closure modeling still remains to be determined, the solutions have demonstrated an ability to correctly account for blade row interactions. Figure 12 shows the computed steady, total temperature field within the four blade rows of the SSME fuel turbine. While supercomputers represent the only possibility for performing many "leading edge" CFD calculations, the high cost of supercomputers and the need to share supercomputing resources among large numbers of users make supercomputers unavailable and/or impractical for many applications. Fortunately, a growing number of computer manufacturers have begun to combine parallel architectures and state-of-the-art microprocessors to form a new class of machine the minisupercomputer. Minisupercomputers, employing 10's (and even 100's) of 
processors, can be purchased for less than $\$ 1 M$, delivering 10 to 20 Mflop performance. It is now possible for many more groups of researchers and departments to become involved in the application of parallel processing to propulsion CFD and to begin to deal with the issues of scaling (i.e., adding processors to gain computing speed for a given problem). A number of studies are currently underway using both shared-memory and distributed-memory minisupercomputers to solve ICFM algorithms [4-5].

A three-pronged approach is being used at NASA Lewis to apply parallel processing to ICFM. First, supercomputers continue to be used to study techniques for parallel processing of large codes. Second, a minisupercomputer is being purchased and will be available, via the ERBNET, in late CY88. The commercial minisupercomputer will provide an excellent training ground for researchers working to adapt existing algorithms and/or develop new algorithms for parallel processing. Finally, a reconfigurable, parallel processing workstation, called the "Hypercluster," is being constructed and is due to be operational in early CY89 [6]. The Hypercluster combines distributed memory (hypercube interconnections) and shared-memory (bus connections at each hypercube node) to provide a unified, cost-effective means of implementing and testing a variety of simulator configurations, and of tailoring a simulator architecture to match a particular algorithm. The initial Hypercluster configuration will be two-dimensional with four nodes and four processors at each node ( Fig. 13).

As illustrated in Fig. 13, the Hypercluster will provide an opportunity to extend parallel processing of the average-passage turbomachinery model beyond 1-processor/blade row. Several approaches are being considered. In one, each cluster of four processors would be devoted to a particular blade row 
calculation. This raises the question: "How best do you divide up the blade row calculations among the four processors?"

In the case of an explicit algorithm (i.e., where each grid point solution only depends on past values at neighboring grid points), partitioning of the blade row solution grid may not be necessary. Milner has proposed a "time partitioning" scheme in which each processor is calculating the entire flow field but at a different time step (or iteration) [7]. At the point where a processor has completed the calculation of the first three columns (or rows) of grid points, another processor begins calculating the next solution step for the grid. Obviously, one of the key advantages of this technique is that the algorithm does not have to be partitioned for parallel solution. The code is merely replicated on each processor. Near-linear speedups are possible as processors are added. Initial tests of the technique have been run on a 2processor CRAY X-MP with a measured speedup of 1.87. This technique and others are being investigated with the aim of demonstrating "optimum" combinations of algorithms and architectures for important ICFM applications.

\section{MULTI-STAGE COMPRESSOR FLOW PHYSICS PROGRAM}

A number of the advanced computational technologies that have been discussed are being focused on the NASA Lewis Multi-Stage Compressor Flow Physics Program. The objective of that program is to increase the fundamental understanding of multi-stage compressor flow physics in order to develop validated, practical methods for computing the flows in multistage compressor systems. Key elements in this program are the continued development of flow models (the average-passage model and a Reynolds-averaged, Navier-Stokes model) and the conducting of multi-stage compressor experiments to collect data that can be used to further define the models. 
Figure 14 illustrates the planned use of parallel processing to link and enhance both the multi-stage CFD development and the fundamental physical experiments. The ERBNET local-area network will provide the connectivity and high-speed data communications required to allow researchers to acquire, share, and compare analytical and experimental results at their desks and in the test cell. If parallel processing can produce 1 to 2 hour turnaround times for running multi-stage CFD codes, the experimentalist will be able to use the codes during the course of a day's running to explain observations and to predict the outcome of the next run.

Laser anemometry is the measurement method of choice at Lewis for obtaining high resolution flow data in high-speed rotating machines [8]. Lewis is recognized as a world leader in the development and application of computercontrolled laser anemometer systems (Fig. 15) for compressor and turbine applications. Parallel processing techniques are being developed that will overcome the long measurement and data reduction times associated with laser measurements of the three-dimensional flow fields. This will allow the researcher to watch the evolution of flow field displays as the data are collected and to detect anomolies in the data, repeating or adjusting the experiment if necessary, without lengthy delays.

\section{CONCLUDING REMARKS}

The NASA Lewis Research Center is exploiting advances being made in computational technology to develop propulsion system simulations that may eventually be capable of accurately predicting the performance, weight, cost, and reliability of propulsion components, subassemblies, and systems before hardware is built. The development of this simulation capability and its practical application to propulsion system design will be one of the "grand challenges" to be faced in aerospace propulsion during the next decade [9]. Through its 
relationships with the propulsion companies, computer vendors, other research laboratories, and academia, NASA is working to ensure that the computing needs of the propulsion community are adequately addressed and that the required blends of hardware and software technologies are made ready to respond to those needs.

The development of a numerical propulsion system simulator also depends on being able to integrate many disciplines (e.g., computational fluid mechanics, computational structural mechanics, mathematics, computer science, and computer engineering) to produce the required analytical models and codes. It also requires the development of techniques for effectively calculating, visualizing, and analyzing the results. This paper has described a number of recent initiatives that have led to the formation of multi-disciplinary research teams and the establishment of a new computational infrastructure and methodology for ICFM research. The combination of these resources will lead to an acceleration of progress toward the goal of more efficient, reliable, and durable propulsion systems.

\section{REFERENCES}

1. Miller, B.A.; Anderson, B.H.; and Szuch, J.R.: Internal Fluid Mechanics Research on Supercomputers for Aerospace Propulsion Systems. NASA TM-100289, 1988.

2. Williams, A.D.: The Development of an Intelligent Interface to CFD FlowSolver Codes. To be presented at the Symposium on Advances and Trends in Computational Structural Mechanics and Fluid Dynamics, Washington, D.C., Oct. 17-19, 1988. (NASA TM- , to be published). 
3. Mulac, R.A., et al.: The Utilization of Parallel Processing in Solving the Inviscid Form of the Average-Passage Equation System for Multistage Turbomachinery. AIAA Paper 87-1108, June 1987.

4. Modiano, D.: Performance of a Common CFD Loop on Two Parallel Architectures. MIT CFD Laboratory Report CFDL-TR-87-11, Nov. 1987.

5. Smith, W.A.: Multi-grid Solution of Euler Equations. PhD Thesis, Cornell University 1987.

6. Blech, R.A.: The Hypercluster: A Parallel Processing Test-Bed Architecture for Computational Mechanics Applications. NASA TM 89823, 1987.

7. Milner, E.J.; Blech, R.A.; and Chima, R.V.: Time-Partitioning Simulation Models for Calculation on Parallel Computers. NASA TM-89850, 1987.

8. Strazisar, A.J.; and Powell, J.A.: Laser Anemometer Measurements in a Transonic Axial Flow Compressor Rotor. J. Eng. Power, vol. 103, no. 2, Apr. 1981, pp. 430-437.

9. A Research and Development Strategy for High Performance Computing. Executive Office of the President, Office of Science and Technology Policy, Nov. $20,1987$.

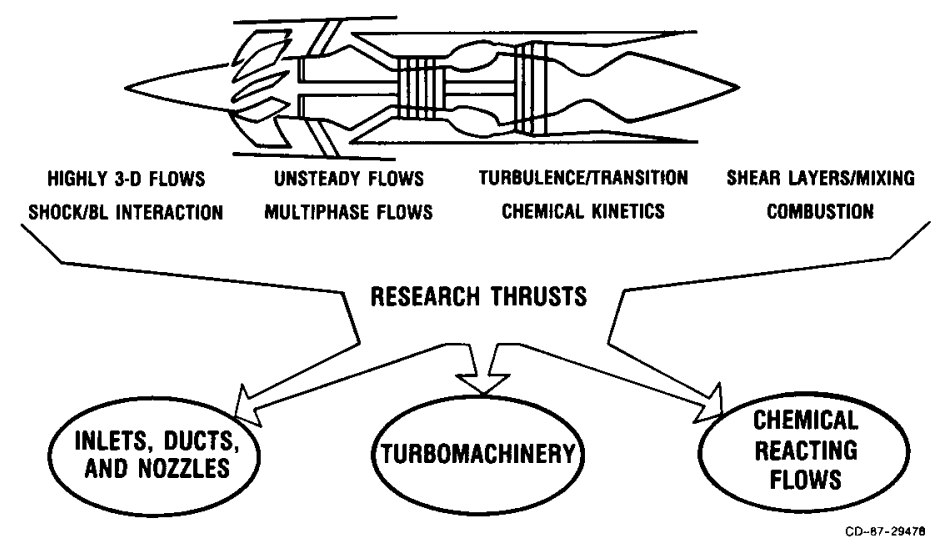

FIGURE 1. - RESEARCH THRUSTS IN INTERAL COMPUTATIONAL FLUID MECHANICS.

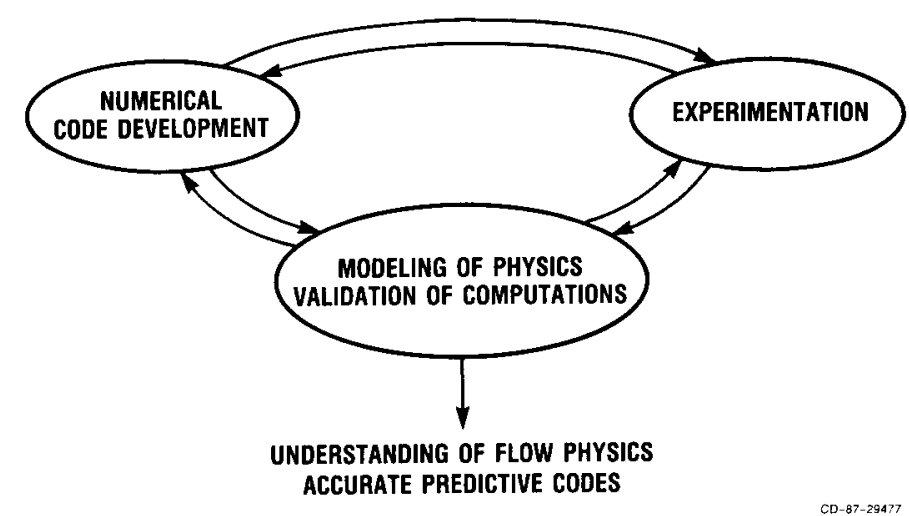

FIGURE 2. - CLOSELY COUPLED EXPERIMENTAL AND COMPUTATIONAL RESEARCH. 


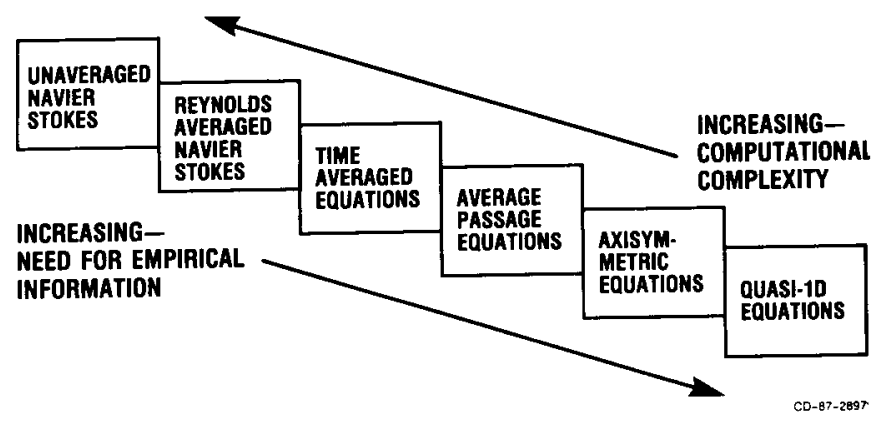

FIGURE 3. - LEVELS OF COMPLEXITY FOR COMPUTATIONAL ANALYSIS

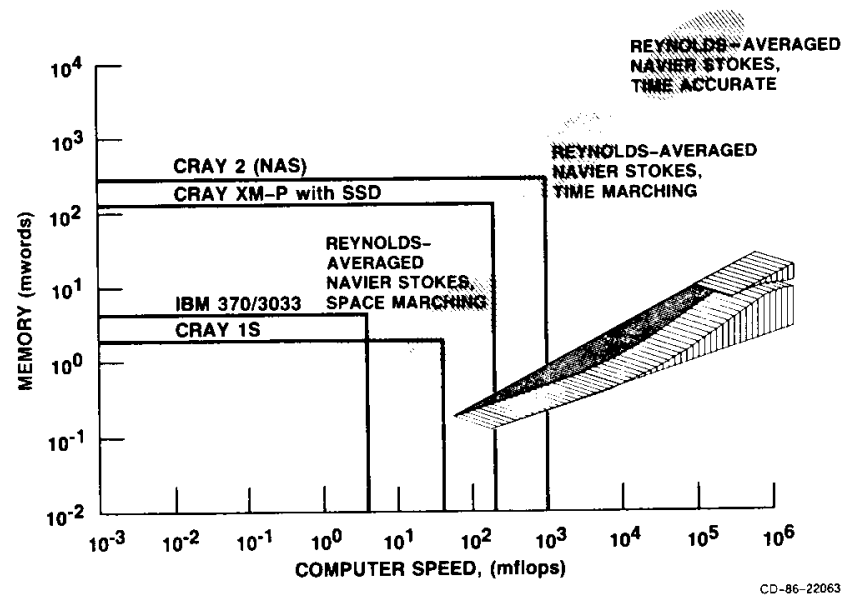

FIGURE 4. - COMPUTER CAPABILITIES AND REQUIREMENTS FOR 3D HYPERSONIC INLET CALCULATIONS.

\section{TECHNOLOGIES \\ - SUPERCOMPUTERS \\ - PARALLEL PROCESSORS \\ - EXPERT SYSTEMS \\ - INTERACTIVE 3-D GRAPHICS \\ - NETWORKS \\ - DBMS \\ - SOFTware engineERING}

IMPROVED CODES

- MORE ACCURATE

- easier to USE

- VALIDATED BY EXPERIMENTS

- PROVIDE NEW PHYSICAL INSIGHTS

- BASIS FOR BETTER, FASTER DESIGNS

FIGURE 5 - FUTURE IMPACT OF TECHNOLOGY ON INTERNAL COMPUTATIONAL FLUID MECHNANICS.

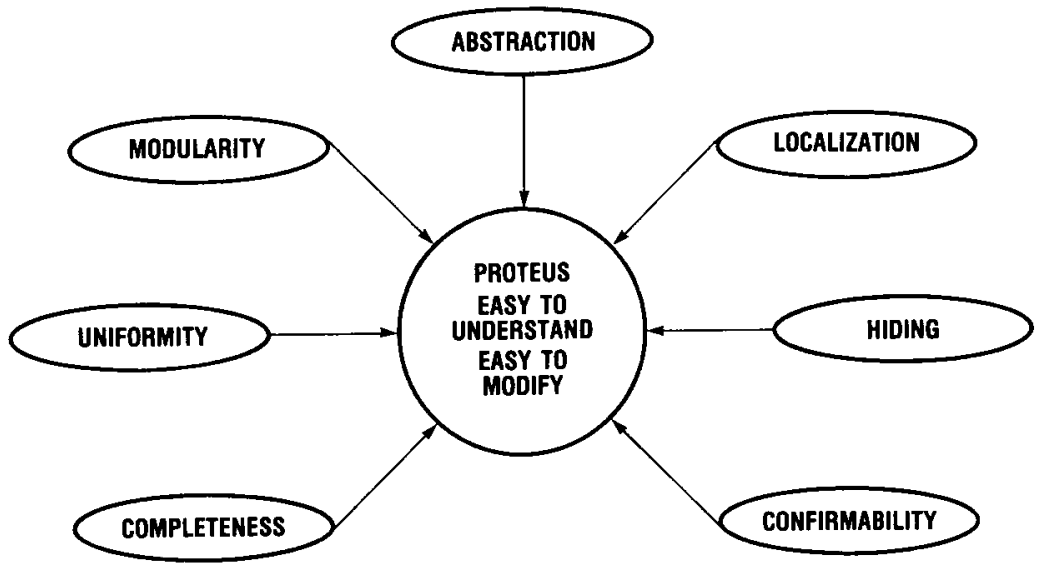

FIGURE 6. - SOFTWARE ENGINEERING PRINCIPLES APPLIED TO PROTEUS CODE DEVELOPMENT. 


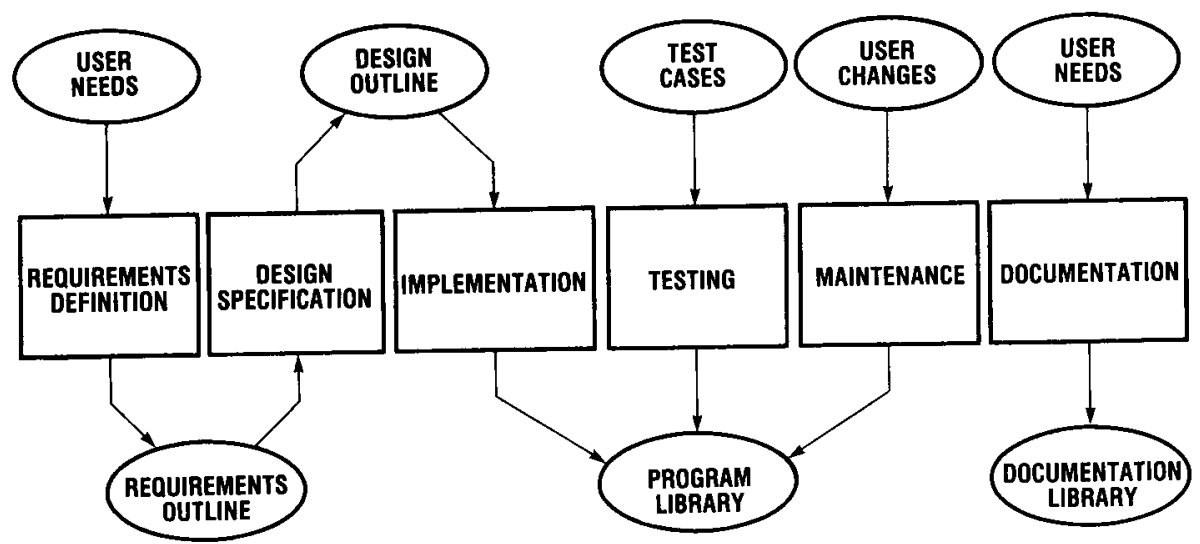

FIGURE 7. - PROTEUS CODE DEVELOPMENT CYCLE.

ORIGINAL PAGE IS

OE RDOR QUALITY

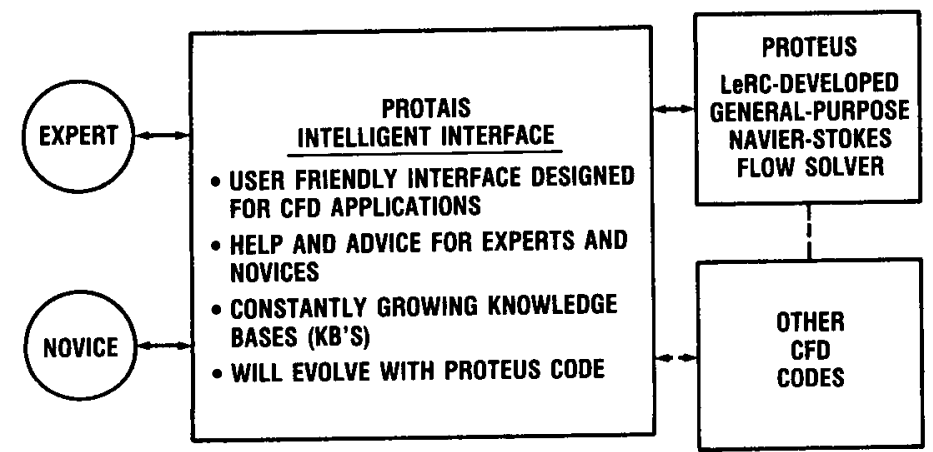

FIGURE 8. - APPLICATION OF ARTIFICIAL INTELLIGENCE TO COMPUTATIONAL FLUID DYNAMICS.

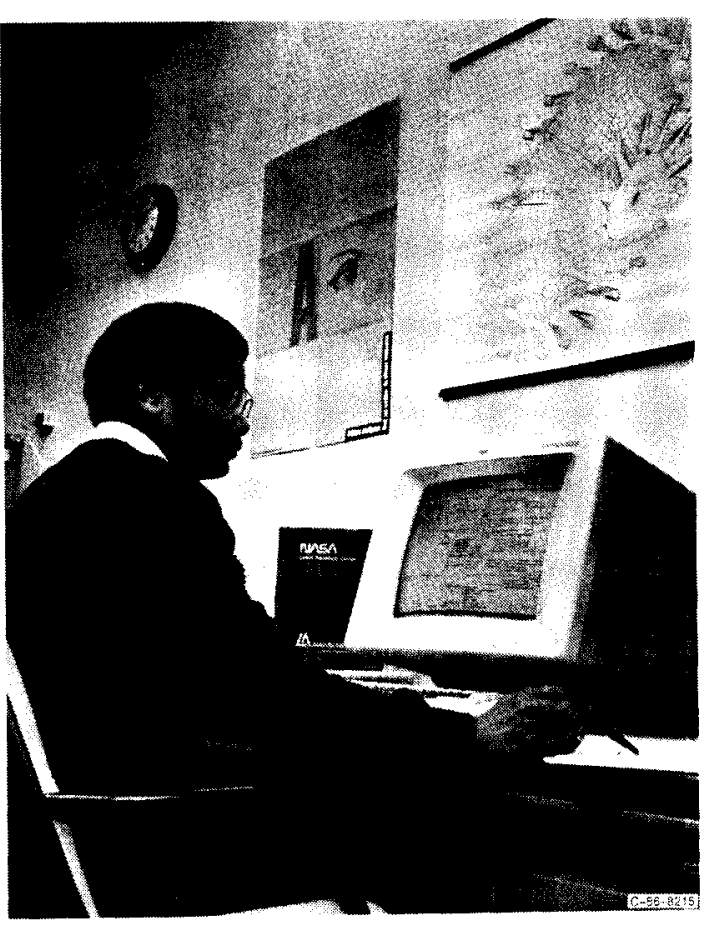

FIGURE 9. - ADVANCED INTELLIGENT WORKSTATIONS. 


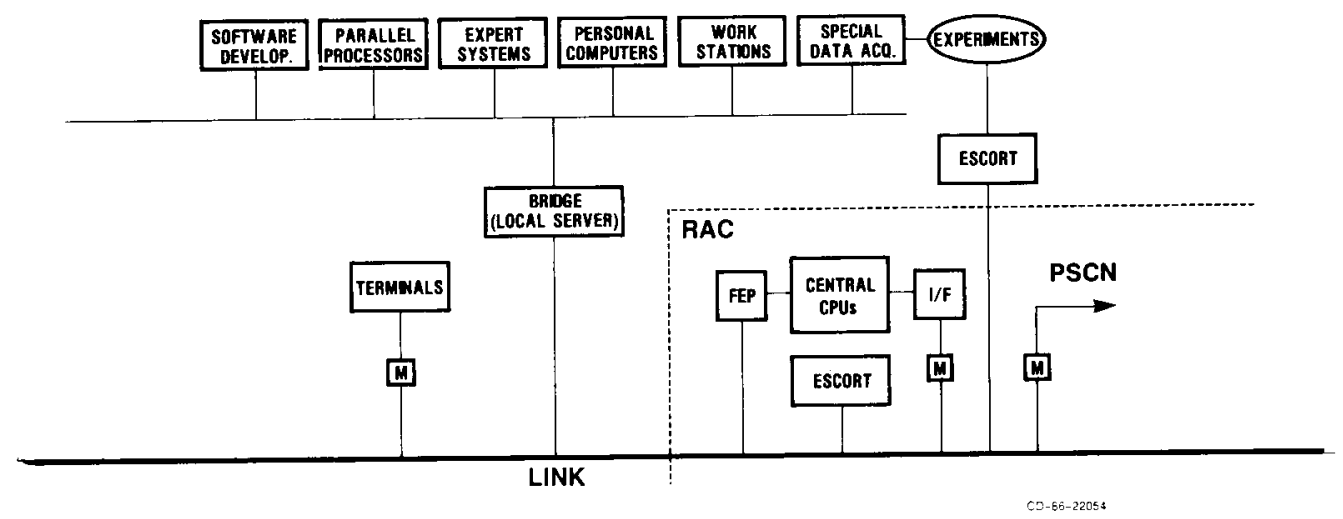

FIGURE 10. - ERBNET LOCAL AREA COMPUTING NETWORK.

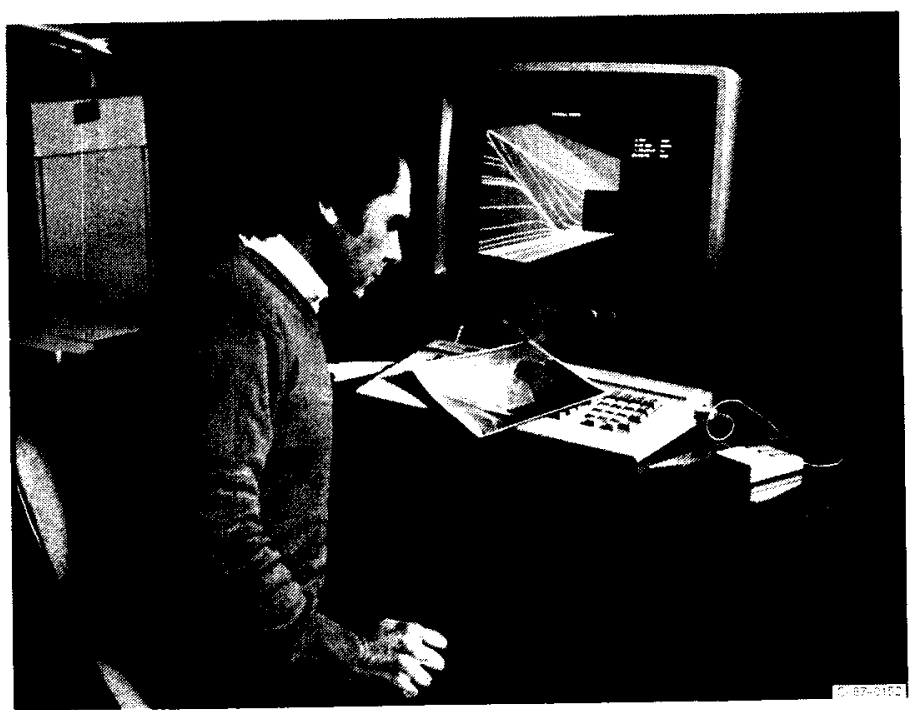

FIGURE 11. - GRAPHICS WORKSTATIONS FOR VISUALIZATION OF 3D FLOW FIELD DATA.

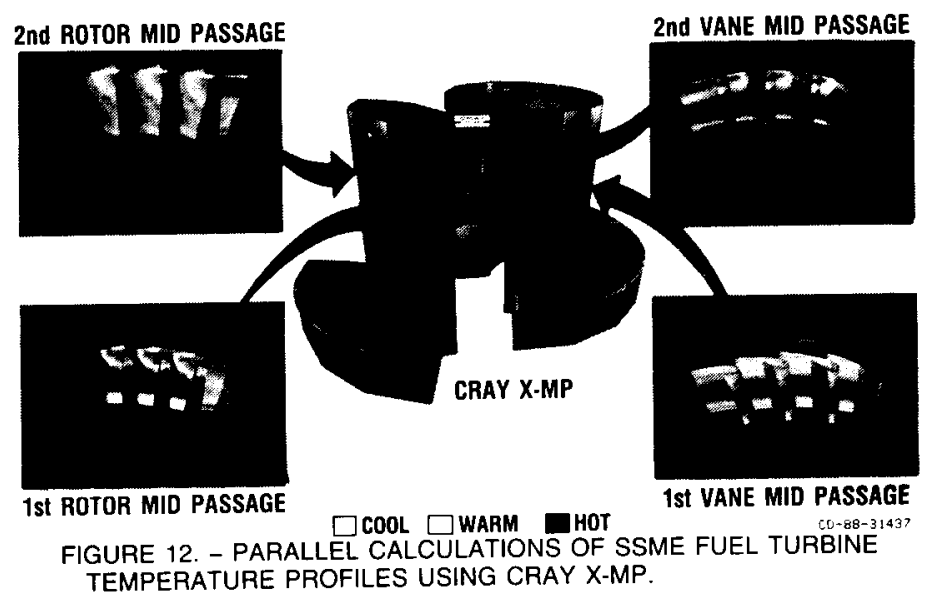




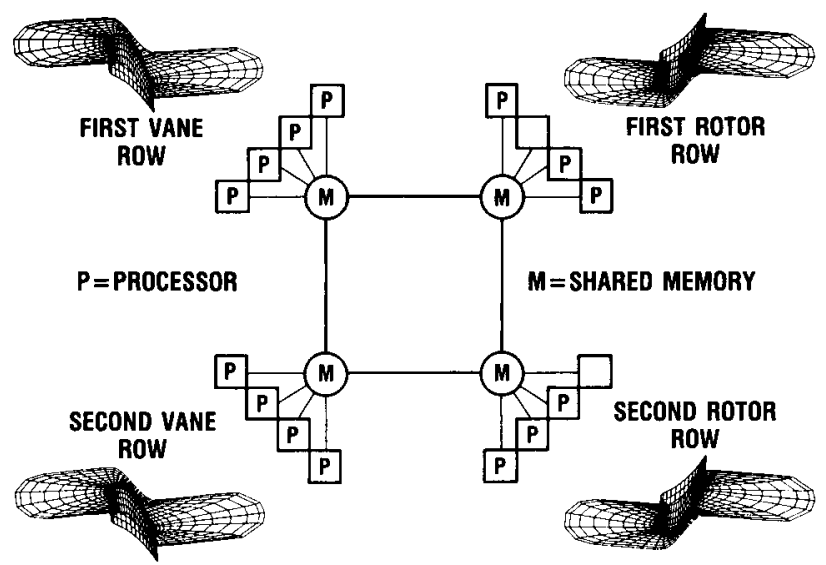

FIGURE 13. - MULTISTAGE TURBOMACHINERY ANALYSIS USING THE HYPERCLUSTER WORKSTATION (4-NODE, 16 PROCESSOR CONFIGURATION).
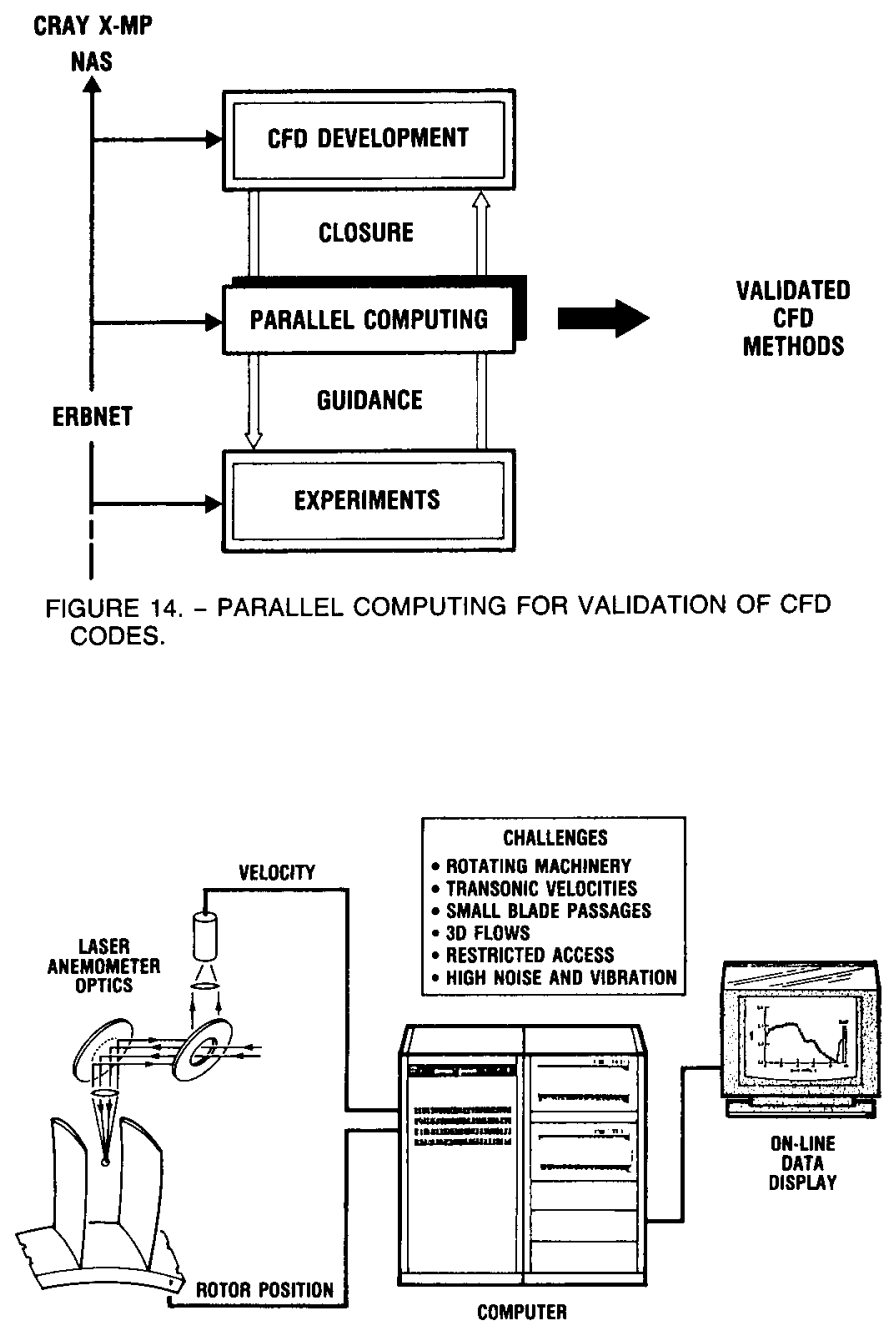

FIGURE 15. - TURBOMACHINERY LASER ANEMOMETRY SYSTEMS. 


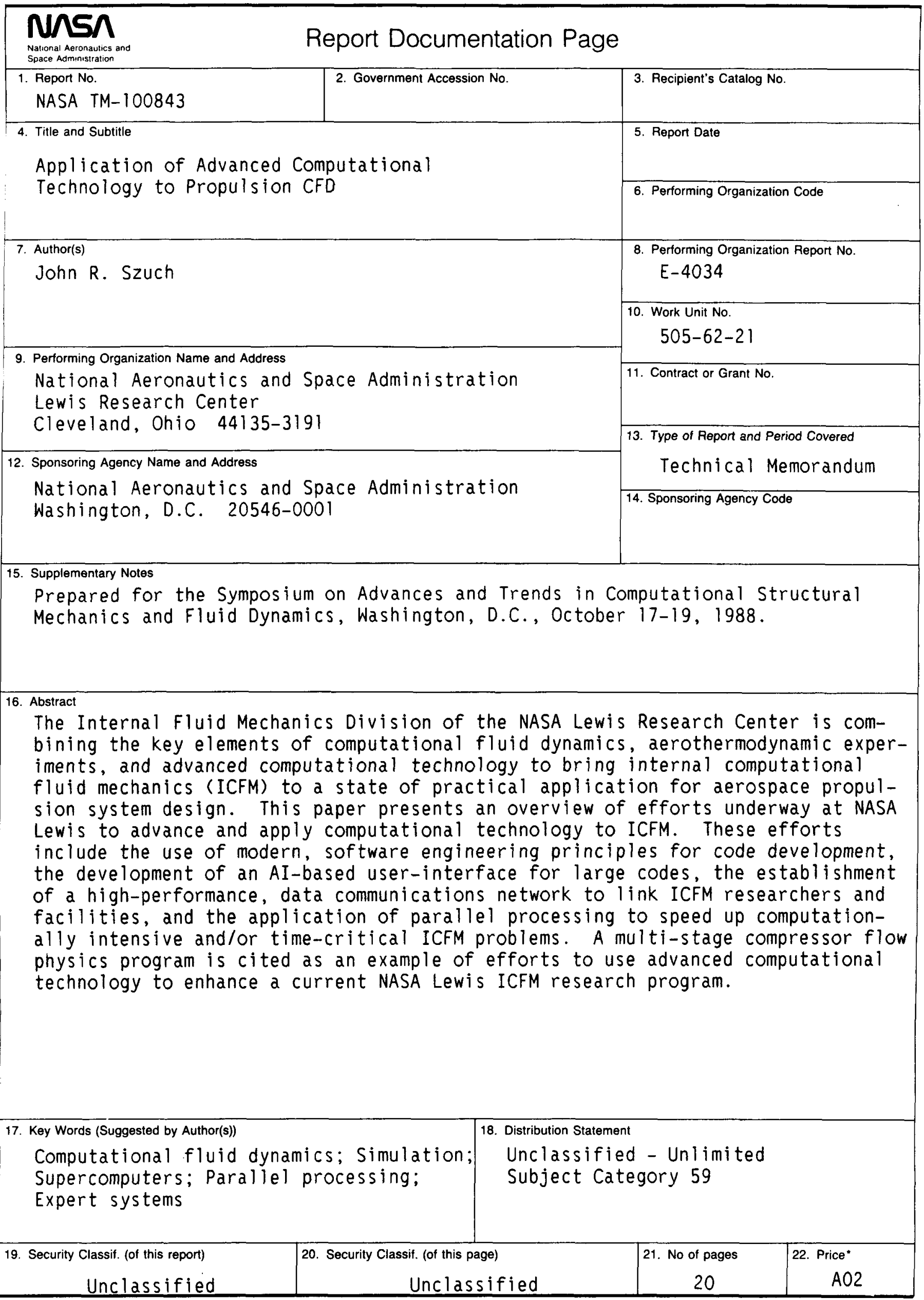

